\title{
Consilium Smartphone App for Real-World Electronically Captured Patient-Reported Outcome Monitoring in Cancer Patients Undergoing anti-PD-L1-Directed Treatment
}

\author{
Andreas Trojan ${ }^{a, b} \quad$ Urs Huber $^{a} \quad$ Mathis Brauchbar ${ }^{c} \quad$ Ulf Petrausch $^{a}$ \\ aOnkoZentrum Zürich, Zurich, Switzerland; bUniversity of Zurich, Zurich, Switzerland; \\ cmobile Health AG, Zurich, Switzerland
}

\section{Keywords}

Smartphone app · Electronically captured patient-reported outcomes · Common

Terminology Criteria for Adverse Events · Immunotherapy · Cancer

\begin{abstract}
Digital patient monitoring gains importance for quality of clinical cancer care. Our case report provides insight into usability and acceptance of a smartphone app for monitoring of electronically captured patient-reported outcomes in patients undergoing immunotherapy. During 3 months, 6 patients with advanced or metastatic PD-L1-positive cancer of the lung, prostate, and bladder who underwent checkpoint immunotherapy were using the Consilium app for standardized and structured electronic reporting of symptoms and therapy side effects. We evaluated the number and quality of symptom entries as well as usability and safety of shared reporting between the patient and the treating physician. Duration of anti-PD-L1-directed immunotherapy in the 6 patients ranged from 4 to 10 months and comprised a total of 21 anti-PD-L1-directed immunotherapy cycles. Patients reported between 4 and 16 different symptoms, of which the most frequent (57\%) were dry cough, fatigue, shortness of breath, fever, and appetite loss. Overall, 1,279 symptom entries were counted, corresponding to 2.4 symptom entries per patient per day. Symptom severity grading ranged from 0.1 (very slight symptoms) to 7.8 (severe symptoms), which triggered prespecified alerts in 4 of the 6 patients. No unplanned visits were noted, and no safety issues occurred. Satisfaction with the app usability was high, as was the beneficial effect on consultation. Usability and reviewed data entries indicate high shared reporting efforts of patients and treating physicians and overall satisfaction with electronically reported patient outcomes.


Trojan et al.: Consilium Smartphone App for ePRO Monitoring in Cancer Patients

\section{Introduction}

Patient-reported outcomes (PROs), such as symptoms and functional status, are commonly measured in clinical trials. There is growing interest to integrate electronically captured PROs (ePROs) into routine clinical practice during chemotherapy and immunotherapeutic interventions [1]. We previously reported on the efficacy of the Consilium app in breast cancer patients for improved communication between patient and physician and patient wellbeing through continuous and structured recording of patients' treatment and related side effects and symptoms [2]. The app is intended to continuously allow oncologists to monitor the progress of patients' symptoms, since ePROs create symptom progression charts based on structured patient entries and also notify a patient to contact the treatment center in case symptoms are out of acceptable range. In addition, doctors are asked to share information on symptom grading, as defined in the Common Terminology Criteria for Adverse Events (CTCAE) standards, with their patients. Consequently, others have shown that PROs not only improve symptom management, but also allow for the reduction of emergency admissions and improve the patient's quality of life, potentially prolonging cancer survival [1]. Few studies so far have reported on PROs in non-small-cell lung cancer and, in particular, on impact of digital patient monitoring on quality of clinical care of cancer immunotherapy [3-5]. This present report of 6 cases was part of a larger multi-centric observational study on electronically reported symptoms and therapy side effects during the course of outpatient cancer care (NCT03578731). It explores on real-world e-health usability data on a small number of patients undergoing checkpoint inhibitor blockade with respect to number and quality of electronically reported symptoms, usability satisfaction, and safety aspects.

\section{Material and Methods}

ePROs with the patients' personal mobile device and the Consilium app were collected during 3 months in patients with advanced or metastatic PD-L1-positive cancer who initiated immunotherapy. Patients had previously given informed consent to the observational study for electronic PRO monitoring (https://clinicaltrials.gov/ct2/show/NCT03578731) that will analyze the utility and reliability of self-reported electronic symptom monitoring in more detail. Patients assessed here were chemotherapy-naïve or had received one prior chemotherapy regimen and initiated checkpoint inhibitor therapy. Anti-PD-L1 checkpoint blockade was conducted according to recommendations from the institutional tumor board and performed according to the manufacturer's instructions. Patients then electronically entered symptoms in a structured and standardized manner (according to the CTCAE 4.0 criteria) during a 3-month observation period. Alerts were generated automatically by the system

Table 1. Patient demographics/treatment

\begin{tabular}{lllllll}
\hline $\begin{array}{l}\text { Age, } \\
\text { years }\end{array}$ & Gender & Site & Stage & Metastasis & Previous TXT & $\begin{array}{l}\text { Duration anti-PD-L1, } \\
\text { months }\end{array}$ \\
\hline 48 & $\mathrm{~m}$ & prostate & IV & bone, lymph nodes & radiation & 4 \\
66 & $\mathrm{~m}$ & lung & III & adjuvant & cisplatin/Alimta & 4 \\
72 & $\mathrm{~m}$ & lung & III & adjuvant & carboplatin/Taxol & 10 \\
59 & $\mathrm{~m}$ & lung & IV & lung & na & 4 \\
69 & $\mathrm{~m}$ & lung & IV & lung & carboplatin/Etopophos & 9 \\
71 & $\mathrm{~m}$ & urothel & IV & lymph nodes & na & 4 \\
\hline
\end{tabular}


Table 2. Symptom gradings and alerts

\begin{tabular}{lllllll}
\hline $\begin{array}{l}\text { Symptom } \\
\text { entries }\end{array}$ & $\begin{array}{l}\text { Different } \\
\text { symptoms }\end{array}$ & $\begin{array}{l}\text { Grade } \\
\text { min. }\end{array}$ & $\begin{array}{l}\text { Grade } \\
\text { max. }\end{array}$ & $\begin{array}{l}\text { Alerts } \\
\text { triggered }\end{array}$ & $\begin{array}{l}\text { Unplanned } \\
\text { visits }\end{array}$ & Reviews \\
\hline 31 & 6 & 0.5 & 3.7 & no & no & 2 \\
115 & 10 & 0.1 & 7.9 & 2 & no & 3 \\
273 & 16 & 0.1 & 6.5 & 3 & no & 3 \\
458 & 9 & 0.1 & 7.8 & 7 & no & 3 \\
145 & 6 & 0.1 & 4.6 & no & no & 4 \\
257 & 4 & 0.1 & 6.5 & 2 & no & 2 \\
\hline
\end{tabular}

when the patient entered side effects that exceeded concerning and predefined grades. Here, assessment of Consilium usefulness was estimated on a small number of subjects via an exit survey. The primary endpoint of this study was the assessment of the number, characteristics, and intensity of electronically reported symptoms and therapy side effects during the first 3 months of checkpoint inhibition, with the intention to rate numbers and quality of electronically reported symptoms with respect to grading, alerts, and safety issues.

\section{Results and Discussion}

ePROs were recorded and analyzed in patients undergoing checkpoint inhibitor therapy from March to October 2019 in 4 patients with advanced lung cancer and high PD-L1 status, 1 patient with prostate cancer (TMB unknown), and another with urothelial cancer. Patients were aged 47-72 years (median 62 years), all patients were male and suffered from metastasis either in lymph nodes, bones, or lungs. One patient had concurrently experienced pelvic irradiation therapy for bone metastasis, while 3 others had received chemotherapy and the 2 remaining had no other previous systemic interventions at all (Table 1). Duration of checkpoint inhibition was between 4 and 10 months. Altogether, 1,279 symptom entries were recorded. Numbers of symptom data entries from the 6 patients ranged from 31 to 458 within the 3-month period (Table 2) with a young prostate cancer patient providing exceptionally sparse information. On average, 2.4 data entries were performed every day, and patients overall reported between 4 and 16 different symptoms (range $0.3-5$; median 8.8 ). From a 100 -point severity scale $(0.1$ reflecting very slight symptoms to 10.0 reflecting very strong, unbearable symptoms), the analyzed 6 patients undergoing immunotherapy graded the symptoms severity from 0.1 to 7.9 (Table 2). This corresponded to a CTCAE grading between grade 0 and grade 3, a methodology that is described in our previous study in more detail [2]. Upon the predefined symptoms severity, 4 of the 6 patients also triggered a total of 14 alerts, all of which correlated to cough, respiratory stress, fever, and fatigue, respectively (Table 2), and made patients aware of making contact with their treating center. As immunotherapies are variable from case to case and hard to predict, they also generate dysimmune toxicities, while some of the above-mentioned symptoms are likely to be associated with immunerelated adverse events. These mainly involve the gut, skin, endocrine glands, liver, and lung and can negatively affect the individual outcome, thus indicating a need for timely reporting. The most frequent symptoms from all 6 patients ranked according to the number of entries are displayed in Figure 1. Overall, the alerts in this small cohort did not result in any unplanned hospitalizations. However, due to alerts generated from the mobile device app, 6 of the alerts resulted in telephone consultations with the treating center or oncologist on call, which in 
Trojan et al.: Consilium Smartphone App for ePRO Monitoring in Cancer Patients

Fig. 1. Most frequent symptoms ranked according to number of entries.

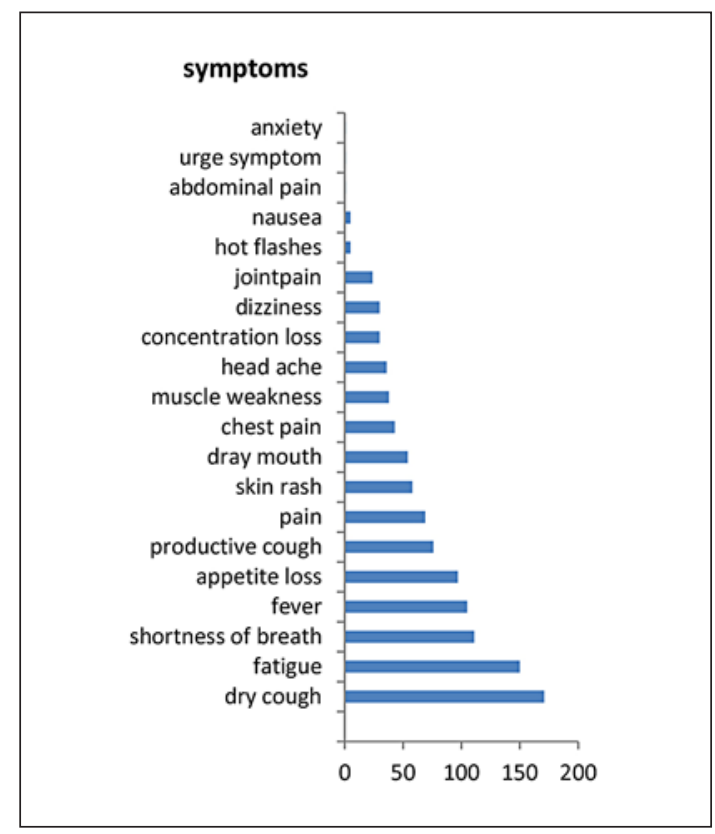

Table 3. Usability and usefulness of the smartphone app (total patients $n=67$ )

Answers

I find the app helpful

The app is easy to use

The app helps me deal with the symptoms of my illness

The app has had a positive effect on doctor visits

My records were taken into account by the doctor during consultations

My symptoms are taken seriously by the doctor

I believe that my personal data will be treated confidentially and used securely

I would recommend the app to other patients
Good/very good
$91 \%$
$98 \%$
$76 \%$
$80 \%$
$89 \%$
$97 \%$
$100 \%$
$97 \%$

turn caused early blood controls and ambulatory clinical assessments in 3 patients during regular office hours. In particular, the shared reporting may indicate both the high collaborative motivation in immunotherapeutic strategies as well as awareness of study conduct. On average, 3 planned review sessions were performed with the treating physician, which also served to conduct the required review of symptom progression charts. Assuming that the graphical display of the relevant symptoms and early reaction to side effect management occurred timely [6], this potentially would have corresponded to a $20 \%$ reduction of unnecessary admissions to acute admission units.

Since we were interested in exploring the usability and comfort of use of the Consilium app, we also performed a survey of 80 consecutive patients undergoing treatment within the larger multi-centric observational study on self-reported symptoms (NCT03578731). The analysis of the first 67 consecutive patients (84\%) who had completed the final questionnaire in week 12 demonstrated that a great majority of these patients reported good or very good effects of the app on symptom management, doctor consultation, and an efficient communication together with a high satisfaction with respect to usability of the app. More than $95 \%$ of users reported on favorable quality of shared reporting, and $97 \%$ of users would recommend the Consilium app to other patients (Table 3). 
When compared to a more comprehensive feedback from patients and treating personnel using the STAR system, the same proportion of patients (98\%) who completed the exit satisfaction survey found STAR easy to use; $84 \%$ found it useful; and $82 \%$ would recommend it to other patients. Despite positive feedback from patients, clinical personnel found that the STAR system increased their current workload without enhancing patient care [7,8]. However, the program helped many patients feel more empowered in their postoperative recovery so that they would recommend its use. In contrast, a positive impact of digital patient monitoring on quality of clinical care of cancer immunotherapy-treated patients with advanced/metastatic non-small-cell lung cancer, encompassing a weekly symptom questionnaire, was reported with respect to patient empowerment, time-saving in symptom reporting, and efficient communication as well as decreased need for telephone consultation [5]. Time to deterioration in 5 symptoms and a composite of 3 symptoms (cough, dyspnea, and chest pain) and PRO data were collected using EORTC QLQ-C30 and LC13 questionnaires. Similar to the Consilium app, raw scores were standardized to a 0 - to 100 -point range, with a $\geq 10$-point score change defined as clinically meaningful. While time to deterioration was defined as the time from randomization until confirmed clinically meaningful deterioration (i.e., a $\geq 10$-point score change from baseline) [2], in our study, a grade 3 or worse symptom automated a trigger for alerts. In most questionnaires and PRO settings, missing data remain a concern, but backup data collection strategies can bring self-report compliance rates up to about $85 \%$ in unselected routine care patients with advanced cancers [4, 9].

In summary, there is a strong rationale for collecting PRO measures, such as symptoms and functional status, all of which are commonly recorded in clinical trials [7, 8]. Increasing interest in integrating electronically captured PROs into routine clinical practice seems both tempting and challenging. Patient self-empowerment and self-reporting should improve patient-clinician communication, symptom detection, and symptom control [2]. Patientreported data may be used for quality assessment as well as in comparative effectiveness research. However, employing strictly electronic recording of PROs with an app on the patient's smartphone has not yet been extensively explored. Alerts as well as graphic displays of structured and standardized outcome data entries are currently evaluated for trustworthiness in a larger prospective clinical trial in order to demonstrate that patient self-reporting in routine care enhances quality of care and patient satisfaction and is expected to become more common in the future. The American Society for Clinical Oncology (ASCO) has several ongoing initiatives to develop standards in this area. Once barriers to implementation, such as logistics, interfacing, data presentation and interpretation, as well as costs, have been largely overcome, data savings for predictive analysis of each case can begin.

\section{Statement of Ethics}

Published research complies with the guidelines for human studies and was conducted ethically in accordance with the World Medical Association Declaration of Helsinki. The authors state that all subjects have given their written informed consent to publish their case. The study protocol (NCT03578731) was approved by the local ethical committee on human research.

\section{Disclosure Statement}

A.T. and M.B. are initiators and stock owners of mobile Health AG, a startup company that operates the Consilium smartphone app. A.T. serves as a chief medical officer and M.B. as chief financial officer for the startup company. 


\section{Funding Sources}

This study on case reports, the preparation of data, and the manuscript were not funded. However, conduct of the international study (NCT03578731) was sponsored by the Foundation Swiss Tumor Institute Zürich, Switzerland.

\section{Author Contributions}

A.T., U.H., and U.P. are physicians and contributed to the conduct of the study and to writing of the case reports. M.B. contributed to the study design and writing of the manuscript.

\section{References}

1 Basch E, Deal AM, Kris MG, Scher HI, Hudis CA, Sabbatini P, et al. Symptom monitoring with patient-reported outcomes during routine cancer treatment: a randomized controlled trial. J Clin Oncol. 2016 Feb;34(6):55765.

2 Egbring M, Far E, Roos M, Dietrich M, Brauchbar M, Kullak-Ublick GA, et al. A mobile app to stabilize daily functional activity of breast cancer patients in collaboration with the physician: a randomized controlled clinical trial. J Med Internet Res. 2016 Sep;18(9):e238.

3 Pérol M, Pavlakis N, Levchenko E, Platania M, Oliveira J, Novello S, et al. Patient-reported outcomes from the randomized phase III ALEX study of alectinib versus crizotinib in patients with ALK-positive non-small-cell lung cancer. Lung Cancer. 2019 Dec;138:79-87.

4 Iivanainen S, Alanko T, Peltola K, Konkola T, Ekström J, Virtanen H, et al. ePROs in the follow-up of cancer patients treated with immune checkpoint inhibitors: a retrospective study. J Cancer Res Clin Oncol. 2019 Mar; 145(3):765-74.

5 Schmalz 00, Jacob C, Ammann J, Liss B, Iivanainen S, Kammermann M, et al . Impact of digital patient monitoring (DPM) on quality of clinical care of cancer immunotherapy (CIT)-treated patients with advanced/metastatic non-small cell lung cancer. Ann Oncol. 2019 Dec;30(Suppl 11):xi30-xi31.

6 Snyder C, Smith K, Holzner B, Rivera YM, Bantug E, Brundage M. Making a picture worth a thousand numbers: recommendations for graphically displaying patient-reported outcomes data. Qual Life Res. 2019 Feb;28(2): 345-6.

7 Basch E, Leahy AB. Reporting standards for patient-reported outcomes in clinical trial protocols and publications. J Natl Cancer Inst. 2019 Apr 11;111(11):1116-7.

8 Basch E, Dueck AC. Patient-reported outcome measurement in drug discovery: a tool to improve accuracy and completeness of efficacy and safety data. Expert Opin Drug Discov. 2016 Aug;11(8):753-8.

9 Basch E. High compliance rates with patient-reported outcomes in oncology trials submitted to the US Food and Drug Administration. J Natl Cancer Inst. 2019 May 1;111(5):437-9. 